Milena Anđelić*

Faculté de Philologie

Université de Belgrade
UDK: 811.133.1'36:821.133.1-31.09

Rabelais F.

DOI: $10.19090 /$ gff.2021.3.71-85

originalni naučni rad

\title{
LE DONT CAUSATIF DANS LES ROMANS DE RABELAIS ET AUJOURD'HUI
}

Dans cet article, nous parlons de l'ancien sens causatif du pronom relatif dont, qui a connu un essor particulier au $\mathrm{XVI}^{\mathrm{e}}$ siècle. Nous avons choisi pour corpus les cinq romans de Rabelais. Le dont causatif avait la particularité d'avoir très souvent comme antécédent la proposition principale entière. La proposition principale avait la valeur de cause, alors que la proposition relative introduite par dont fonctionnait comme conséquence. Ce sont le plus souvent les verbes de sentiment de la proposition subordonnée qui donnaient la possibilité d'utiliser le relatif dont de sens causatif. La fréquence du sens causatif de dont comparé aux autres sens possibles de ce relatif (origine, moyen, manière...) était plus élevée à l'époque et compte presque $50 \%$ d'occurences, malgré la possibilité de chevauchement avec les sens d'origine et de propos. Les exemples extraits de l'original comparés aux traductions en français moderne ont montré non seulement quelles expressions ont remplacé le dont causatif de nos jours (à cause de, par la suite de...) mais aussi qu'il est possible de garder ce corrélatif pour créer un effet stylistique plus archaïsant à l'intérieur d'un texte littéraire.

Mots clés : dont, relation cause-conséquence, diachronie, Rabelais.

\section{INTRODUCTION}

Dans cet article, nous allons analyser les effets de sens du pronom dont dans les cinq romans de Rabelais (Gargantua, Pantagruel, Le Tiers Livre, Le Quart Livre, Le Cinquième Livre) et nous concenter sur l'effet de sens causatif. Le sens causatif est intéressant à analyser parce qu'il s'agit d'un effet de sens du pronom relatif dont qui n'est plus utilisé de nos jours, alors qu'il était très fréquent autrefois, et surtout au XVIe siècle.

Les romans de Rabelais ont été choisis comme corpus pour plusieurs raisons. Il s'agit d'un corpus extensif stylistiquement plus ou moins unifié (même auteur,

\footnotetext{
*milenandelic@hotmail.com
} 
même sujet) et par son ancienneté susceptible à contenir un nombre satisfaisant d'exemples du pronom dont, et particulièrement de dont de sens causatif. D'un autre côté, la syntaxe de la phrase rabelaisienne est assez proche de celle de nos jours pour que, dans les traductions, il soit possible dans la majorité des cas de garder la même structure syntaxique et le même ordre des mots - ce qui nous aide à vérifier si dont aussi peut garder sa place originelle ou si le traducteur doit utiliser une autre expression à sa place.

Dans la première partie de l'article, nous allons brièvement exposer les origines du pronom relatif dont et son évolution diachronique. Ensuite, dans la deuxième partie, nous expliquerons quels sont les différents sens possibles de ce pronom et comment les analyser. Dans la troisième partie, nous exposerons la fréquence ce tous ses sens dans un tableau pour montrer que c'est bien le sens causatif qui est le plus représenté dans les romans de Rabelais. Puis, nous définirons le phénomène de la causalité et de quelle manière elle est exprimée par le pronom dont. Dans l'avant-dernière partie, nous comparerons les exemples des romans de Rabelais avec la situation en français contemporain en consultant les traductions de G. Demerson (Gargantua seulement) et M-M. Fragonard (œuvre intégrale). Nous tenterons aussi de répondre à la question s'il est possible de reprendre le dont causatif tel quel en français contemporain. Enfin, nous exposersons quelques difficultés d'analyse, à savoir la fusion possible du sens causatif avec le sens d'origine d'une part, et, d'autre part, le sens causatif avec le sens de propos.

Les exemples sont tous cités en référence à l'édition intégrale bilingue de Marie-Madeleine Fragonard.

\section{ORIGINES DE DONT}

Le pronom relatif dont est issu du latin vulgaire, du composé pléonastique deunde (< de + unde), qui, traduit littéralement, donne «de d'où » (Brachet, 1868 : 179 ; Moignet, 1976 : 45 ; Drašković, 1977 : 58, 108 ; Picoche, 1979 : 75 ; Zinc, 1994 : 101). Avec le temps, en passant du latin classique au latin vulgaire, on a ressenti le besoin de renforcer le pronom interrogatif unde par la préposition de (Nyrop, 1979 : 54). En français, dont était d'abord utilisé comme pronom interrogatif direct (exemple 1) ou indirect ayant un sens spatial d'origine (tout comme son étymon), avant de se spécialiser aussi pour l'emploi relatif (exemple 2) créant probablement le même effet de sens que 
l'interrogatif (Haase, 1898 : 95 ; Brunot, 1933 : 231 ; Moignet, 1976 : 171; Popović, 2014 : 238). Nous remarquons que dont en tant qu'interrogatif et en tant que relatif coexsistent toujours au XVIe siècle, au temps de Rabelais.

(1) Dont vient cela, messieurs ? pensez y, je vous pry (Rabelais, $2017: 280$ ).

(2) Je vous remectz à la grande chronicque Pantagrueline recongnoistre la genealogie et antiquité dont nous est venu Gargantua (Rabelais, 2017 : 164).

$\mathrm{Au} \mathrm{IX}^{\mathrm{e}}$ siècle, dans la Séquence de Sainte Eulalie, on retrouve la première attestation littéraire de dont, où le sens et l'emploi ont déjà évolué. Dont n'est plus spatial (exemple 3), il est utilisé comme pronom relatif.

(3) Il li enortet dont lei nonque chielt / Qued elle fuiet lo nom christiien (Anđelić, $2015: 10$ ).

En effet, outre le sens (spatial) d'origine, dont reprend aussi tous les autres sens de la préposition de qui le compose : la cause, le moyen, le propos et autres (Brunot, 1933 : 231 ; Moignet, 1976 : 162, 171, 290 ; Popović, 2014 : 243). L'emploi spatial, qui rappelle le plus l'origine de dont en tant qu'adverbe de lieu (provenant du latin unde), devient de plus en plus rare dès la fin du moyen-âge (Foulet, 1930 : 295 ; Nyrop, 1979 : 54) avant d'être définitivement remplacé par d'où. Néanmoins, on retrouve sporadiquement cet emploi même au XVIIIe siècle chez Voltaire.

(4) Ma vie est dans les camps dont vous m'avez tiré (Brachet, 1868 : 180).

\section{EFFETS DE SENS}

Comme tous les pronoms, le relatif dont est un mot déictique, ce qui veut dire que, si l'on veut déterminer les effets de sens qu'il peut avoir, il est impossible de le faire hors contexte. À l'intérieur de la phrase, le sens lui est attribué par :

1. la sémantique de son antécédent (un syntagme nominal ou la proposition principale entière) ;

2. le verbe ou l'adjectif dont il est le complément (dans la proposition relative) ; 
3. l'énoncé dans son intégralité.

Nous avons déjà vu les exemples de l'effet de sens d'origine (1, 2 et 4), attribué principalement à dont par le verbe de la relative (venir) et souvent un antécédent dénotant l'idée d'origine (un lieu ou la généalogie). Le sens de moyen ou instrument (5) lui vient de la combinaison de la sémantique de l'antécédent et du verbe de la relative, que l'on peut paraphraser avec l'expression au moyen de, alors que le sens de propos (6) dépend des verbes de parole et de pensée.

(5) A beaulx gouvetz qui sont petitz demy cousteaux dont les petitz enfans de nostre pays cernent les noix (Rabelais, 2017 : 288).

(les couteaux au moyen desquels ils cernent les noix)

(6) [...] genealogie de Gargantua, plus entiere que nulle aultre. Exceptez celle du messias, dont je ne parle (Rabelais, 2017 : 166).

(parler de la généalogie)

Le sens de manière (7) vient seulement de l'antécédent «la manière » ou un synonyme, alors que l'effet de sens "génitif» (8) dépend de la structure de l'expression dont le pronom dont fait partie (Anđelić, 2015 : 41-51).

(7) (manière) Gargantua venu considera la contenence du moyne : et la forme dont il pendoit (Rabelais, $2017: 340$ ).

(8) (génitif partitif) Les nouvelles de ces oultraiges feurent sceues par toute l'arme, dont plusieurs commencerent murmurer contre Picrochole (Rabelais, 2017 : 362).

(plusieurs d'entre eux)

L'effet de sens de la matière (9) provient de l'antécédent, lui-même désignant un matériau, et des verbes du type «faire, construire, fabriquer».

(9) (matière) les coustelettes, dont on faict en Pygmion les beaulx petits arcs (Rabelais, 2017 : 948). 
(10) (cause) Ce que considerant Alexandre en l'hippodrome [...], advisa que la fureur du cheval ne venoit que de frayeur qu'il prenoit à son umbre. Dont, montant dessus, le feist courir encontre le Soleil (Rabelais, 2017 : 222).

L'effet de sens de la cause (10) qui nous intéresse particulièrement dans cet article, est déduit à partir de l'énoncé entier, de la partie «cause » de la proposition principale et de la partie "conséquence» de la proposition relative, reliées par dont, soit à l'intérieur d'une même phrase, soit séparées.

\section{LA FRÉQUENCE}

En comptant la fréquence de chaque effet de sens à travers les cinq romans de Rabelais, nous avons relevé que le sens causatif compte presque $50 \%$ d'occurences, suivi du sens spatial de l'origine (20\%), du moyen (11\%), du génitif $(8 \%)$, du propos $(4 \%)$, de la matière $(1,5 \%)$ et la manière $(0,5 \%)$. Dans le tableau ci-dessous, nous avons indiqué le nombre d'occurences de chacun de ces sens par roman.

Tableau 1 : Tableau des fréquences

\begin{tabular}{|c|c|c|c|c|c|c|c|c|c|}
\hline & \multicolumn{9}{|c|}{ effets de sens } \\
\hline roman & $\begin{array}{c}\mathbf{n}^{\mathbf{0}} \text { total } \\
\text { d'occurences }\end{array}$ & origine & moyen & matière & manière & cause & propos & génitif & autre \\
\hline Garg. $^{\mathbf{1}}$ & 53 & 9 & 5 & $/$ & 1 & $27(3)$ & 2 & 3 & 3 \\
\hline Pant. $^{\mathbf{1}}$ & 86 & 21 & 12 & $/$ & $/$ & $24(11)$ & $/$ & 12 & 6 \\
\hline TL $^{\mathbf{1}}$ & 18 & 1 & 1 & $/$ & $/$ & $9(1)$ & 5 & $/$ & 1 \\
\hline $\mathbf{Q L}^{\mathbf{1}}$ & 29 & 6 & 1 & 2 & $/$ & 14 & $/$ & $/$ & 6 \\
\hline CL $^{\mathbf{1}}$ & 14 & 4 & $/$ & 1 & $/$ & 7 & 1 & 1 & $/$ \\
\hline total & 200 & 41 & 19 & 3 & 1 & $81(15)$ & 8 & 16 & 16 \\
\hline
\end{tabular}

Les exemples que nous avons classés dans la colonne « autre » (8\%), sont les occurences de dont où il est complément des verbes de sens spécifique, propre à eux-mêmes, qu'on ne peut pas aisément classer avec d'autres verbes de sens similaire (comme c'est le cas par exemple pour les verbes de parole). Il s'agit donc des verbes comme sauver, contrepoincter, louer, avoir faute de.

1 Explication des abréviations: Garg. (Gargantua), Pant. (Pantagruel), TL (Le Tiers Livre), QL (Le Quart Livre), CL (Le Cinquième Livre). 
Il y a aussi un certain nombre d'occurences à l'interprétation ambiguë, où l'on peut hésiter entre le sens causatif et le sens de propos : nous avons néanmoins classé ces exemples dans la catégorie «cause » avec leur nombre entre parenthèses. Nous allons commenter ces exemples dans la partie 6 de notre article et expliquer d'où vient l'ambiguïté.

Il est tout aussi intéressant de noter que le nombre d'occurences total pour chacun des romans est très différent et ne dépend aucunement de la longueur du roman, mais seulement de son contenu.

\section{LA CAUSALITÉ ET L'EFFET DE SENS CAUSATIF DANS LE CAS DE DONT}

Les phénomènes de cause et de conséquence ne peuvent être définis séparément. En effet, nous appelons une cause un événement à partir duquel d'autres événements découlent logiquement. La conséquence, en prenant la direction opposée, est un événement qui se produit en réponse à quelque chose qui s'est produit auparavant. Ainsi, on ne peut parler ni d'une cause sans conséquence, ni d'une conséquence sans cause présumée. Grivaz affirme la même chose lorsqu'elle attribue au lien causal le caractère de « contrefactualité » : « si la cause ne s'était pas produite, il n'y aurait pas eu de conséquence » (Grivaz, $2009: 182$ ).

Puisqu'il ne s'agit évidemment que d'une différence de point de vue, cette situation a conduit certains chercheurs à abandonner au moins formellement ces divisions traditionnelles et à commencer à traiter la relation de cause à effet indépendamment de la disposition des éléments qu'elle relie. Alors Rossari, au lieu du nom classique de cette connexion, utilise le terme « implication» (Rossari, 1996 : 272). En effet, la question n'est plus de savoir ce que cette connexion relie, mais comment ; tandis que, d'un autre côté, il est très important de savoir le contenu sémantique des arguments de « cause » et de « conséquence » afin qu'ils puissent être identifiés comme tels.

Dans son article, Jacques Moeschler (Moeschler, 2003) énumère et classe les critères de détermination de la relation causale. Il distingue des aspects linguistiques et les aspects pragmatiques :

1. Les aspects linguistiques des relations de cause à effet : 
a) proximité spatio-temporelle (la contiguïté),

b) asymétrie temporelle.

2. Les aspects pragmatiques, qui donnent plus d'espace à l'interprétation subjective :

a) la possibilité de déterminer un lien plus fort ou plus faible d'inférence causale (la contingence),

b) l'influence du contexte (les conditions ceteribus partibus),

c) l'existence de règles (la généralité).

En d'autres termes, pour que deux arguments soient interprétés comme cause et effet, ils doivent appartenir à une partie similaire de la réalité, qu'une connexion logique s'établisse entre eux, avec l'impératif que la cause précède temporellement et logiquement la conséquence. À ce propos, Grivaz note bien que le lien causal n'exclut pas le lien temporel (Grivaz, 2009 : 178, 179). Nous dirons même qu'il l'impose.

Moeschler donne un exemple de connexion forte (insoluble) d'inférence causale (Moeschler, $2003: 16$ ) :

(11) Brutus a tué César. César est mort.

Il s'agit presque d'une tautologie. La prémisse « cause » de la première phrase conduit sans équivoque à «effet» de la seconde. À l'opinion de Moeschler, l'implication est ici sémantique. Cependant, dans un autre exemple du même auteur (12), la conséquence offerte est possible, mais pas nécessaire.

(12) Max a poussé Jean. Jean est tombé (Moeschler, 2003 : 16).

Ici Moeschler affirme que l'implication est pragmatique et que l'inférence causale est plus faible.

En ce qui concerne le pronom relatif dont de sens causatif, la cause est exprimée par l'antécédent - soit un syntagme nominal soit, plus souvent, la proposition principale entière - et la conséquence se retrouve dans la proposition relative. 
Dans le tableau 2, nous avons repris l'exemple 10 cité plus haut: le cheval avait peur de son ombre (cause) et par conséquent (effet), Alexandre le fait courir dans le sens où il lui est impossible de la voir :

Tableau 2: Analyse d'exemple

[...] la fureur du cheval ne venoit que de frayeur qu'il prenoit à son umbre.

proposition principale - antécédent sens causatif
Dont, montant dessus, [Alexandre] le feist courir encontre le Soleil.

proposition relative

sens consécutif

- dont reprend le sens causatif

Le relatif dont qui relie ces deux phrases a le sens causatif, malgré le fait qu'il se trouve à l'intérieur de la proposition exprimant la conséquence. C'est dû au fait que, en tant que pronom, il reprend naturellement le sens de son antécédent. Ici nous avons aussi l'exemple d'une situation fréquente chez Rabelais et dans les œuvres du XVIe siècle en général : c'est la possibilité d'introduire dont au début de la phrase consécutive, puisque la phrase causative est terminée par un point. En français contemporain, il n'est plus d'usage de séparer ainsi le relatif de son antécédent.

En ce qui concerne la nature de l'inférence causale, à notre avis il s'agit d'une inférence causale faible à l'origine, renforcée par la présence de dont servant de corrélatif.

Dans la proposition relative, les verbes et les adjectifs de sentiment sont fréquents (Anđelić, 2015 : 31), parce que les sentiments exprimés dans la relative (verbes s'éjouir et avoir dépit des exemples suivants) sont une réaction à une situation dans la principale :

(13) Ainsi croissoit Pantagruel de jour en jour et prouffitoit à veue d'œil, dont son pere s'esjouyssoit par affection naturelle (Rabelais, 2017 : 434).

(14) les marroufles de la vile batoyent les escholiers, dont eut despit (Rabelais, 2017 : 438). 


\section{FRANÇAIS CONTEMPORAIN - TRADUCTIONS/ADAPTATIONS}

Dans le tableau 3 nous avons classé les différentes traductions ou adaptations de dont en français contemporain par roman. Pour Gargantua, nous présentons deux traductions : celle de Guy Demerson (GD) et celle de Marie-Madeleine Fragonard (MMF). Pour les quatre autres romans, il s'agit des traductions de M.-M. Fragonard seulement.

Tableau 3 : Traductions de dont par roman

\begin{tabular}{|c|l|}
\hline Roman & \multicolumn{1}{|c|}{ Traductions/adaptations du sens de cause en français contemporain } \\
\hline Garg. $^{2}$ & $\begin{array}{l}\text { (GD) donc, alors, ce qui [fit, causa, amena], qui, en, c'est pourquoi, pour } \\
\text { cette raison, aussi [+inversion], en conséquence, verbe en conclure } \\
\text { (MMF) d'où, ce dont, ce qui, et, « et il en dit » }\end{array}$ \\
\hline Pant. $^{2}$ & $\begin{array}{l}\text { [antécédent explicité ou non] dont, ce dont, ce qui, que, en, donc, alors, à } \\
\text { cause de, ce pourquoi, et, « après ce récit », (pas de traduction) }\end{array}$ \\
\hline TL2 $^{2}$ & à cause de quoi, d'où, en, ce pourquoi, dont [il advint], c'est ainsi que \\
\hline $\mathbf{Q L}^{2}$ & $\begin{array}{l}\text { ce dont, d'où [il advient], alors, dont [il arriva], construction causative } \\
\text { faire+inf., donc }\end{array}$ \\
\hline $\mathbf{C L}^{2}$ & si bien que, ce qui [+ constr. causative], et \\
\hline
\end{tabular}

Nous pouvons constater que G. Demerson remplace systématiquement le dont causatif par d'autres mots: conjonctions, locutions conjonctives, autres pronoms relatifs et diverses expressions. Alors que M.-M. Fragonard garde plus de formules archaïques et décide souvent de ne pas remplacer le dont causatif, ce qui expliquerait peut-être aussi le fait qu'on nomme cette traduction «adaptation » dans l'édition que nous avons consultée.

Voici quelques exemples où nous pouvons observer la différence d'approche au texte original :

(15) [ils] se rendirent à luy sains et saulves, excepté Eusthenes lequel un des Geans avoit egraphiné quelque peu au visaige [...] Et Epistemon qui ne se comparoit

2 Explication des abréviations: Garg. (Gargantua), Pant. (Pantagruel), TL (Le Tiers Livre), QL (Le Quart Livre), CL (Le Cinquième Livre). 
poinct. Dont Pantagruel fut si dolent qu'il se voulut tuer soymesmes (Rabelais, $2017: 580)$.

(15a) [ils] vinrent à lui sains et saufs, sauf Eusthénès qu'un des géants avait un peu égratigné au visage [...] Et Epistémon qui ne se présentait pas. Ce dont Pantagruel fut si peiné qu'il voulait se tuer lui-même (Rabelais, 2017 : 581).

Nous voyons dans 15a que Fragonard a décidé de laisser dont à sa place originelle en ajoutant seulement $c e$ pour renforcer le lien avec l'antécédent. En outre, il n'est pas impossible de rencontrer dont sans ajout d'antécédent ce (Grevisse, 1980 : 601) dans les œuvres de littérature moderne. Ceci est rarement pratiqué, cette construction médiévale étant tombée en désuétude vers la fin du XVIIe siècle.

(16) Les tripes furent copieuses, comme entendez [...] Mais la grande diablerie à quatre personnaiges estoit bien en ce que possible n'estoit longuement les reserver, car elles feussent pourries. Ce que sembloit indecent. Dont fut conclud qu'ilz les bauffreroient sans rien y perdre (Rabelais, 2017 : 178).

(16a) Les tripes furent copieuses, comme vous vous en doutez [...] Mais là où il y eut bien une diablerie à grand spectacle c'est qu'il n'était pas possible de les mettre longtemps de côté, car elles se seraient avariées, ce qui paraissait inadmissible. Il fut donc décidé qu'on les bâfrerait sans rien en predre (Rabelais, 1996 : 73).

(16b) Les tripes furent copieuses, comme vous le comprenez [...] Mais le cas diabolique fut qu'il n'était pas possible de les mettre longtemps en réserve, car elles auraient pourri. Ce qui ne semblait pas convenable. D'où il fut conclu qu'ils les bâfreraient sans rien en predre (Rabelais, 2017 : 179).

Alors que dans 16a Demerson a choisi de non seulement remplacer dont par donc, conjonction explicitement consécutive, mais aussi de changer de verbe (conclure devient décider), les deux choix étant complémentaires, dans 16b Fragonard garde l'ordre des mots et le verbe de l'original et choisit de traduire dont par d'où, locution qui instaure l'effet de sens non pas seulement causatif, mais qui aussi rappelle le sens spatial d'origine, plus ancien.

Il est tout aussi possible de ne pas traduire dont, c'est-à-dire ne pas expliciter la relation cause-conséquence entre les propositions. Dans ce cas, la 
relation cause-conséquence est ou bien assez forte dans le contexte-même pour que le lecteur puisse la déduire, ou bien supprimée par le traducteur qui a choisi d'autres moyens pour mettre en évidence le sens du texte.

(17) [Pantagruel] fut adverty que une grosse et enorme cloche estoit à Saint Aignan dudict Aurelians, en terre [...] [E]lle estoit tant grosse que par engin aulcun ne la pouvoit mettre seullement hors terre [...] Dont voluntiers encliné à l'humble requeste des citoyens et habitans de la dicte ville, delibera la porter au clochier (Rabelais, 2017 : 444).

(17a) il fut aderti qu'une grosse, énorme cloche était par terre à l'église SaintAignan [...] [E]lle était si grosse que personne ne pouvait la sortir de terre avec une machine [...] Volontiers bienveillant à l'humble requête des citoyens et habitants de la ville, il délibéra de la porter au clocher (Rabelais, 2017 : 445).

Dans l'exemple 17, le sens consécutif de la relative dont delibera la porter au clochier est renforcé par une cause supplémantaire ajoutée à l'intérieur : voluntiers encliné à l'humble requeste des citoyens et habitans de la dicte ville, ce qui justifie l'omission du consécutif qui relierait cette relative à la principale (17a). En effet, l'ajout d'un consécutif serait même pléonastique :

(17b) il fut aderti qu'une grosse, énorme cloche était par terre à l'église SaintAignan. [...] [E]lle était si grosse que personne ne pouvait la sortir de terre avec une machine. [...] Volontiers bienveillant à l'humble requête des citoyens et habitants de la ville, il délibéra *donc de la porter au clocher.

\section{CAUSE OU ORIGINE / CAUSE OU PROPOS - PROBLÈMES D'INTERPRÉTATION}

Dans des exemples contenant certains verbes dans la relative dont le sémantisme est soit proche au sens de mouvement, soit qu'il en est dérivé (le verbe advenir de l'exemple 18), on peut remarquer que l'interprétation est à cheval entre celle de l'origine et celle de la cause. Nous tenterons d'expliquer cela par le fait que l'effet de sens de cause dans le cas de dont est dérivé, ou issu, du sens premier d'origine. Comme si, en parlant de la cause de quelque chose, il en revenait presque au même que d'en parler de l'origine. 
(18) Sus ses vieulx jours, il espousa la fille du baillif Concordat, jeune, belle, frisque, gualante, advenante, gratieuse, par trop, encers ses voisins et serviteurs. Dont advint, en succession de quelques hebdomades, qu'il en devient jalous comme un tigre (Rabelais, 2017 : 772).

Ceci est loin d'être explicitement le cas dans la majorité des exemples que nous avons analysés, mais le verbe advenir, à notre avis, situe l'énoncé à peu près vers le milieu du continuum interprétatif origine-cause.

Nous avons relevé un autre problème d'interprétation qui concerne les effets de sens de cause et de "propos » lorsque dont est le complément des verbes de parole (dire, demander, répondre...) et de pensée. S'agit-il alors d'une réaction à une situation dans le contexte précédent ou aux paroles de l'interlocuteur dans un dialogue (favorisant l'interprétation causative) ou s'agit-il d'une simple reprise du sujet de conversation ou du thème de l'énoncé (favorisant l'effet de sens de propos) ?

(19) Le premier que y trouvay, ce fut un bon homme qui plantoit des choulx. Dont tout esbahy luy demanday : " Mon amy, que fais tu icy ? - Je plante, (dist il), des choulx (Rabelais, 2017 : 403).

(20) Le feu mis es fagotz, la flambe feut si grande et si haulte qu'elle couvrit tout le chasteau. Dont penserent que bien tost après la tour seroit arse et demollie (Rabelais, 2017 : 888).

Donc, nous pouvons paraphraser dont des exemples 19 et 20 comme « au sujet de quoi je lui demandai » et «ils en pensèrent » respectivement, si l'on opte pour l'interprétation «propos ». Ou bien, en favorisant l'interprétation causative : «c'est pourquoi je lui demandai» pour l'exemple 19 et «et par conséquent ils pensèrent» pour l'exemple 20. En général, il est impossible de trancher définitivement pour l'une des deux interprétations, et c'est pourquoi nous proposons de les relier. Dans ces exemples, il s'agit bien d'une réaction à ce qui a été fait auparavant avec la reprise du thème établi dans le contexte. 


\section{CONCLUSION}

Dans les cinq romans de Rabelais, le dont causatif est très répandu : presque $50 \%$ d'occurences appartiennent à cet effet de sens.

Si dans la langue française du temps de Rabelais, il était possible d'utiliser d'autres expressions à la place de dont (donc, à quoy [respondit], adoncques, lors), en français contemporain il est désormais obligatoire de faire usage des autres corrélatifs (alors, donc, pour cette raison, c'est pourquoi). Ce sont les solutions que l'on retrouve systématiquement dans la traduction de Guy Demerson. Il est tout aussi possible, dans certains cas, d'omettre le consécutif. Pourtant, il semble que dans les traductions plus archaïsantes où nous sommes tentés de classer celle de M-M. Fragonard, il soit toujours possible de garder le dont causatif pour créer un effet de style.

En ce qui concerne l'analyse-même des exemples de dont à travers l'œuvre de Rabelais, dans certains cas, il est impossible de trancher définitivement s'il s'agit bien du sens de l'origine ou de celui de la cause, les deux se situant sur le même continuum interprétatif. Dans les exemples avec les verbes de paroles et de pensée dans la relative, il y a aussi fusion du sens causatif et du sens de propos.

\section{Milena Anđelić}

THE CAUSATIVE PRONOUN DONT IN THE NOVELS BY RABELAIS AND IN CONTEMPORARY FRENCH

\section{Summary}

In this article, we discuss the ancient causative meaning of the relative pronoun dont, that flourished in the 16th century. We have chosen Rabelais' five novels as our corpus. The causative dont had the particularity of having very often the entire main clause as its antecedent. The main clause had the value of cause, while the relative clause introduced by dont functioned as a consequence. It was most often the sentiment verbs of the subordinate clause that gave the possibility of using the relative in causative sense. The frequency of the causative dont compared to the other senses existing at the time (origin, means, manner...) is higher and counts almost $50 \%$ of occurrences, despite the possibility of overlap with the sense of origin and theme. The examples taken from the original compared to the modern French translations showed not only which expressions have replaced the causative dont nowadays (à cause de, par la suite $d e . .$.$) but also that it is possible to keep this correlative to create a more archaic stylistic$ effect within a literary text.

Key words : dont, the cause-consequence relation, diachrony, Rabelais. 


\section{CORPUS}

Rabelais, F. (1929a). CEuvres complètes de Rabelais. Le tiers livre. Paris : Éditions Fernand Roches.

Rabelais, F. (1929b). CEuvres complètes de Rabelais. Le quart livre. Paris : Éditions Fernand Roches.

Rabelais, F. (1929c). OEuvres complètes de Rabelais. Le cinquiesme livre. Lettres et écrits divers. Paris : Éditions Fernand Roches.

Rabelais, F. (1972). Pantagruel. Paris : Le Livre de Poche.

Rabelais, F. (1996). Gargantua. Paris : Editions du Seuil.

Rabelais, F. (2017). Les Cinq Livres des faits et dits de Gargantua et Pantagruel. Paris : Éditions Gallimard.

\section{RÉFÉRENCES BIBLIOGRAPHIQUES}

Anđelić, M. (2015). Dijahronijski pregled značenja odnosne zamjenice dont (Mémoire de master non publié). Filološki fakultet, Univerzitet $\mathrm{u}$ Beogradu, Beograd. (Анђелић, М. (2015). Дијахронијски преглед значења односне замјенице dont (Mémoire de master non publié). Филолошки факултет, Универзитет у Београду, Београд.)

Brachet, A. (1868). Grammaire historique de la langue française. Paris : Bibliothèque d'éducation J. Hetzel et Cie.

Brunot, F. (1933). Histoire de la langue française des origines à 1900: De l'époque latine à la Renaissance. Paris : Librairie Armand Colin.

Drašković, V. (1977). Uvod u tumačenje starofrancuskih tekstova. Beograd : Naučna knjiga. (Драшковић, В. (1977). Увод у тумачење старофранцуских текстова. Београд : Научна књига.)

Foulet, L. (1930). Petite syntaxe de l'ancien français. Paris : Honoré Champion. Grevisse, M. (1980). Le Bon usage. Paris : Duculot.

Grivaz, C. (2009). Un jeu de règles permettant de déterminer si une relation causale est exprimée entre des propositions. Nouveaux cahiers de linguistique française, 29, 173-195.

Haase, A. (1898). Syntaxe française du XVIIe siècle. Paris : Alphonse Picard et fils.

Moeschler, J. (2003). L'expression de la causalité en français. Cahiers de linguistique française, 25, 11-42. 
Moignet, G. (1976). Grammaire de l'ancien français. Morphologie - Syntaxe. Paris : Klincksieck.

Nyrop, Kr. (1979). Grammaire historique de la langue française. Syntaxe. Particules et verbes. Genève : Slatkine reprints.

Picoche, J. (1979). Précis de morphologie historique du français. Paris : Nathan. Popović, M. (2014). Istorija francuskog jezika. Beograd : Jasen.

Rossari, C. (1996). Les marques de la consécution : propriétés communes et distinctives à la lumière de donc, de ce fait, et il en résulte que. Dépendance et intégration syntaxique, 271-284.

Zinc, G. (1994). Morphologie du français médiéval. Paris : PUF. 
